Doug Geisler, Eva K. Grebel, and Dante Minniti, eds.

\title{
Mass Determinations of Star Clusters
}

\author{
Georges Meylan \\ Space Telescope Science Institute, 3700 San Martin Drive, \\ Baltimore, MD 21218, USA gmeylan@stsci.edu
}

\begin{abstract}
Mass determinations are difficult to obtain and still frequently characterised by deceptively large uncertainties. We review below the various mass estimators used for star clusters of all ages and luminosities. We highlight a few recent results related to (i) very massive old star clusters, (ii) the differences and similarities between star clusters and cores of dwarf elliptical galaxies, and (iii) the possible strong biases on mass determination induced by tidal effects.
\end{abstract}

\section{Introduction}

Open and globular clusters are respectively located, because of their local definition, in the plane and in the halo of our Galaxy. This review will not make any formal distinction between these two kinds of star clusters since (i) the above definitions apply only to our Galaxy and (ii) we do not know if there are any genuine differences in their formation mechanisms. Consequently, we may use the words "open" and "globular", but we shall essentially mean "star clusters", whether they are light or massive, or young or old. This approach is also justified by the fact that, in simulations of the Galactic globular cluster system, the dynamical evolution of an initial mass distribution, of either Gaussian or power-law type, leads always to a predicted distribution consistent with observations: light star clusters dissolve rather quickly, while heavy ones survive longer (Baumgardt 1998, 2001). See also Zhang \& Fall (1999) in the case of the populations of star clusters of the Antennae Galaxies.

Individual masses of star clusters are not easy to measure. As an example, we shall quote the four different mass estimates given for the giant Galactic globular cluster $\omega$ Centauri by Ogorodnikov et al. (1976). From a few stellar radial velocities $\mathcal{M}_{\text {tot }} \simeq 7 \times 10^{5} \mathcal{M}_{\odot}$, from the same radial velocities corrected for the effect of the internal rotation of the cluster $\mathcal{M}_{\text {tot }} \simeq 3 \times 10^{6} \mathcal{M}_{\odot}$, from lowquality proper motions measured on photographic plates $\mathcal{M}_{\text {tot }} \simeq 3 \times 10^{7} \mathcal{M}_{\odot}$ and from gravitational focusing from the same proper motions $\mathcal{M}_{\text {tot }} \simeq 2.8 \times$ $10^{8} \mathcal{M}_{\odot}$. These four different approaches provide results differing by 3 orders of magnitude! Fortunately, things have improved since then, but even the best mass determinations of star clusters remain rather uncertain, typically by a factor of 2 . It is interesting to realize that the age of the Universe, intuitively more difficult to determine than the mass of a nearby star cluster, is more accurately known since the Hubble constant $H_{\circ}$ is now estimated at a better than $20 \%$ level. 


\section{The Giant Galactic Clusters $\omega$ Centauri and 47 Tucanae}

Significant improvements in the quality and numbers of radial velocities have allowed the determinations of reliable mass estimates through the use of various dynamical models. On the one hand, there is the parametric approach. For example, Gunn \& Griffin (1979) developed multi-mass models whose distribution functions $f$ depend on the stellar energy per unit mass $\varepsilon$ and the specific angular momentum $l$. Such models are spherical and have a radial anisotropic velocity dispersion $\left(\overline{v_{r}^{2}} \neq \overline{v_{\theta}^{2}}=\overline{v_{\phi}^{2}}\right)$. They are called King-Michie models and associate the lowered Maxwellian of the King model with the anisotropy factor of the Eddington models:

$$
f(\varepsilon, l) \propto\left[\exp \left(-2 j^{2} \varepsilon\right)-\exp \left(-2 j^{2} \varepsilon_{t}\right)\right] \exp \left(-j^{2} l^{2} / r_{a}^{2}\right)
$$

On the other hand, one never knows which of the assumptions underlying such a model are adhered to by the real system and which are not (Dejonghe \& Merritt 1992). These arguments suggest that it might be profitable to interpret kinematical data from globular clusters in an entirely different manner, placing much stronger demands on the data and making fewer ad hoc assumptions about the distribution function $f$ as well as the gravitational potential $\Phi$. Ideally, the unknown functions should be generated non-parametrically from the data, in an approach pioneered by Merritt $(1993,1996)$. We provide hereafter the results of two studies, parametric and non-parametric, respectively, of the globular cluster $\omega$ Centauri, both studies using exactly the same observational data, viz., the surface brightness profile and 469 stellar radial velocities.

- Parametric: a simultaneous fit of these radial velocities and of the surface brightness profile to a multi-mass King-Michie dynamical model provides mean estimates of the total mass for $\omega$ Cen equal to $\mathcal{M}_{\text {tot }}=5.1 \pm 0.6 \times 10^{6} \mathcal{M}_{\odot}$, with a corresponding mean mass-to-light ratio $\mathcal{M} / L_{V}=4.1$ (Meylan et al. 1995).

- Non-parametric: the potential and mass distribution infered in this method provide a total mass for $\omega$ Cen equal to $\mathcal{M}_{\text {tot }}=2.9 \pm 0.4 \times 10^{6} \mathcal{M}_{\odot}$, with a corresponding mean mass-to-light ratio $\mathcal{M} / L_{V}=2.3$ (Merritt et al. 1997).

\section{Universal Mass-to-Light Ratio?}

With a similar parametric approach applied to the observational constraints obtained for NGC 1835, an old Large Magellanic Cloud globular cluster, KingMichie models give $\mathcal{M}_{\text {tot }}=1.0 \pm 0.3 \times 10^{6} \mathcal{M}_{\odot}$, corresponding to a mean massto-light ratio $\mathcal{M} / L_{V}=3.4 \pm 1.0$ (Meylan 1988, Dubath \& Meylan 1990).

These studies show that when the same kind of dynamical models (KingMichie) constrained by the same kind of observations (surface brightness profile and central value of the projected velocity dispersion) are applied to an old and bright Magellanic globular cluster, viz., NGC 1835, the results seem similar to those obtained in the case of Galactic globular clusters. Consequently, the rich old globular clusters in the Magellanic clouds could be quite similar in mass and $\mathcal{M} / L_{V}$ to the rich globular clusters in the Galaxy. 


\section{The Final Word from Thousands of Stellar Space Motions}

Recently, the amount of data related to stellar motions, viz., radial velocities and proper motions, has increased significantly. In a pioneering ground-based study of $\omega$ Centauri, van Leeuwen et al. (2000) measured the individual proper motions of 7853 probable member stars, from photographic plates with epochs ranging from 1931 through 1935 and 1978 through 1983. An internal proper motion dispersion of 1.0 to $1.2 \mathrm{mas} \mathrm{yr}^{-1}$, equivalent to 25 to $29 \mathrm{~km} \mathrm{~s}^{-1}$ for a distance of $5.1 \mathrm{kpc}$, is found for members near the cluster center. This dispersion decreases to $0.3 \mathrm{mas} \mathrm{yr}^{-1}$, equivalent to $7.5 \mathrm{~km} \mathrm{~s}^{-1}$ in the outer regions. The full dynamical interpretation of these proper motions, combined with a few thousand radial velocities, is in preparation by the same group.

Another group, using HST/WFPC2 images, has obtained slightly better measurements of proper motions for about 15,000 stars in the core of 47 Tucanae, this within a time baseline of only 4 years, with 3 epochs separated by 2 years. See Anderson, King, \& Meylan 1998 for a progress report after the second epoch. These data, combined with the radial velocities of about 5,000 stars, will provide, as in the case of $\omega$ Centauri, an insight into the dynamics of the core of 47 Tucanae, with fundamental by-products such as cluster distance and photometry of variable stars and binaries.

With their new proper-motion techniques and software (Anderson \& King 2000) applied to the HST/WFPC2 archive data for the first epoch and to their own observations for the second epoch, the members of the same group will soon have available similar sets of proper motions for about ten among of the richest and most nearby Galactic globular clusters.

The above studies, with thousands of proper motions and radial velocities constraining dynamical models with three integrals of the motion as well as nonparametric ones, will allow a significant step forward in our understanding of the internal dynamics of massive star clusters.

\section{Mayall II $\equiv$ G1, a Giant Globular Cluster in M31}

Mayall II $\equiv \mathrm{G} 1$ is one of the brightest globular clusters belonging to M31, the Andromeda galaxy. Observations with HST/WFPC2 provide photometric data for the $I$ vs. $V-I$ and $V$ vs. $V-I$ color-magnitude diagrams. They reach stars with magnitudes fainter than $V=27 \mathrm{mag}$, with a well populated red horizontal branch at about $V=25.3 \mathrm{mag}$ (Meylan et al. 2001). From model fitting, that study determines a rather high mean metallicity of $[\mathrm{Fe} / \mathrm{H}]=-0.95 \pm 0.09$, somewhat similar to 47 Tucanae. In order to determine the true measurement errors, Meylan et al. (2001) have carried out artificial star experiments. They find a larger spread in $V-I$ than can be explained by the measurement errors. They attribute this to an intrinsic metallicity dispersion among the stars of G1, which may be the consequence of self-enrichment during the early stellar/dynamical evolutionary phases of this cluster. So far, only $\omega$ Centauri, the giant Galactic globular cluster, has been known to exhibit such an intrinsic metallicity dispersion. This is, a phenomenon certainly related to the deep potential well of each of these two star clusters, which are massive enough to retain the gas expelled by the first generations of very massive stars. 
The structural parameters of G1 are deduced from the same HST/WFPC2 data. Its surface brightness profile provides its core radius $r_{c}=0.14^{\prime \prime}=0.52$ pc, its tidal radius $r_{t} \simeq 54^{\prime \prime}=200 \mathrm{pc}$, and its concentration $c=\log \left(r_{t} / r_{c}\right) \simeq$ 2.5. Such a high concentration indicates the probable collapse of the core of G1. KECK/HIRES observations provide the central velocity dispersion $\sigma_{o b s}=$ $25.1 \mathrm{~km} \mathrm{~s}^{-1}$, with $\sigma_{p}(0)=27.8 \mathrm{~km} \mathrm{~s}^{-1}$ once aperture corrected.

Three estimates of the total mass of this globular cluster can be obtained. The King-model mass is $\mathcal{M}_{K}=15 \times 10^{6} \mathcal{M}_{\odot}$ with $\mathcal{M} / L_{V} \simeq 7.5$, and the Virial mass is $\mathcal{M}_{V i r}=7.3 \times 10^{6} \mathcal{M}_{\odot}$ with $\mathcal{M} / L_{V} \simeq 3.6$. The King-Michie model fitted simultaneously to the surface brightness profile and the central velocity dispersion value provides mass estimates ranging from $\mathcal{M}_{K M}=14 \times 10^{6} \mathcal{M}_{\odot}$ to $17 \times 10^{6} \mathcal{M}_{\odot}($ Meylan et al. 2001).

Table 1. Three mass determinations for Mayall II $\equiv \mathrm{G} 1$ and $\omega$ Cen

\begin{tabular}{ccc}
\hline \hline Mass & $\begin{array}{c}\text { Mayall II } \\
{\left[10^{6} \mathcal{M}_{\odot}\right]}\end{array}$ & $\begin{array}{c}\omega \text { Centauri } \\
{\left[10^{6} \mathcal{M}_{\odot}\right]}\end{array}$ \\
\hline King & 15 & 4.3 \\
Virial & 7.3 & 2.9 \\
King-Michie & $13-18$ & 5.1 \\
\hline
\end{tabular}

The spread between the three mass determination values listed in Table 1 give a better idea than their individual formal (smaller) errors about their true uncertainties. The masses of both clusters are known to about a factor of two. Although not very precise, all of these mass estimates make G1 more than twice as massive as $\omega$ Centauri, the most massive Galactic globular cluster. G1 is unique in M31 because of its projected location $40 \mathrm{kpc}$ away from the center of the galaxy, but there are at least three other bright globular clusters in this galaxy which have velocity dispersions $\sigma_{o b s}$ larger than $20 \mathrm{~km} \mathrm{~s}^{-1}$, implying rather large masses.

\section{On the Origin of the Most Massive Globular Clusters}

Such large masses are related to the metallicity spread the origin of which is still unknown. It may come either (i) from metallicity self-enrichment in a massive globular cluster, (ii) from primordial metallicity inhomogeneity in a binary proto-cluster cloud, followed by early merger, or (iii) from the fact that the present globular cluster is merely the remaining core of a previously larger entity, e.g., originally a dwarf galaxy subsequently pruned by dynamical evolution.

Although $\omega$ Centauri is the best studied globular cluster, because of its size and relative proximity $(\sim 5.1 \mathrm{kpc})$, many conundrums remain: viz., (i) the metallicity spread among stars (Freeman \& Norris 1981), (ii) its double Main Sequence (Anderson 1997), (iii) the different kinematics between metal-rich and -poor stars (Norris et al. 1997), and (iv) a correlation between metallicity and age, implying that this cluster enriched itself over a timescale $\sim 3$ Gyr (Hughes \& Wallerstein 2000 and Hilker \& Richtler 2000). 
Table 2. Parameters for the massive globular cluster Mayall II $\equiv \mathrm{G} 1$ and the nucleus of the dwarf elliptical galaxy NGC 205 (see Meylan et al. 2001)

\begin{tabular}{ccc}
\hline \hline Parameters & Mayall II & NGC 205 \\
\hline$\sigma_{p}(0)$ & $27.8 \mathrm{~km} \mathrm{~s}^{-1}$ & $30 \mathrm{~km} \mathrm{~s}^{-1}$ \\
$M_{V}$ & $-10.94 \mathrm{mag}$ & $-9.6 \mathrm{mag}$ \\
$r_{c}$ & $0.52 \mathrm{pc}$ & $0.35 \mathrm{pc}$ \\
$\mu(0, V)$ & $13.47 \mathrm{mag} / \operatorname{arcs}^{2}$ & $12.84 \mathrm{mag} / \operatorname{arcs}^{2}$ \\
\hline
\end{tabular}

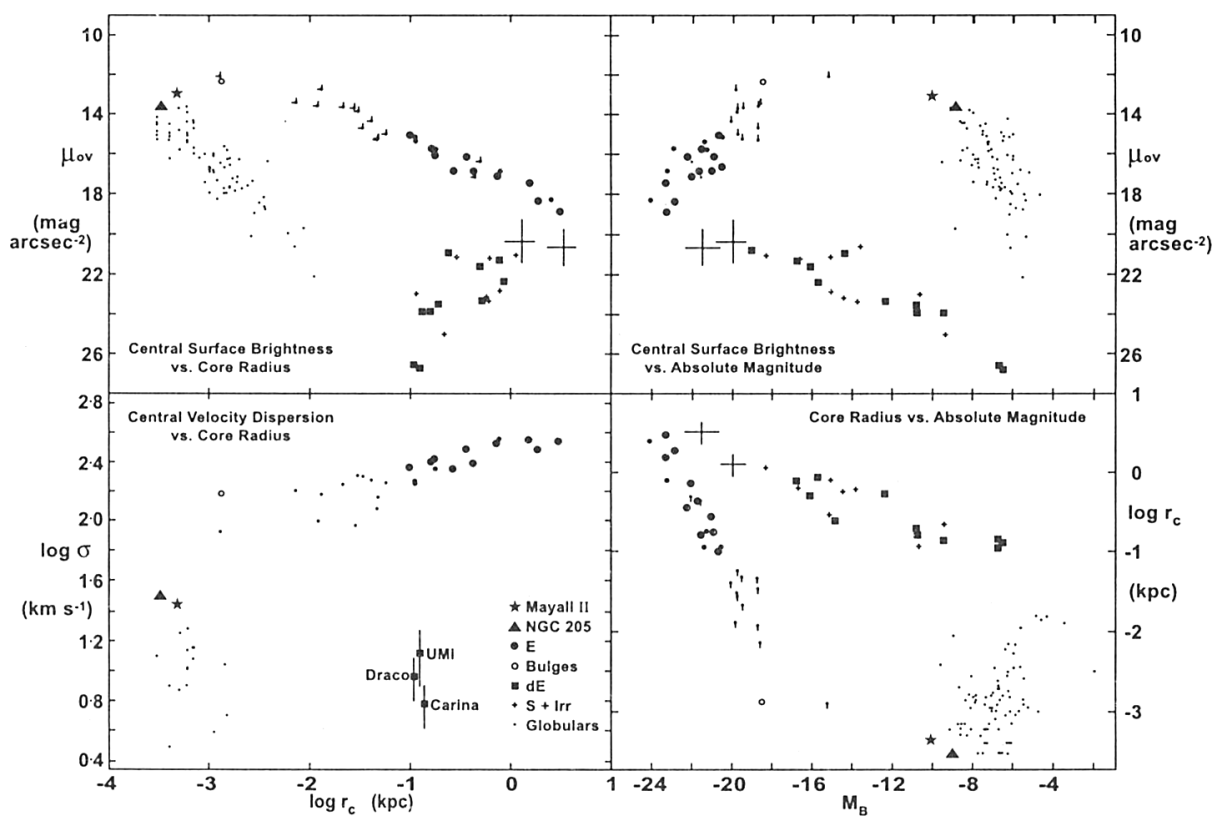

Figure 1. Reproduction of Fig. 3 from Kormendy (1985) in which we have added the positions, using the four parameters in Table 2, of Mayall II $\equiv$ G1 (star) and of the nucleus of NGC 205 (triangle). These fall in all four panels right on the sequences defined by globular clusters and always away from the sequences defined by ellipticals, bulges, and dwarf ellipticals. 
Let us consider (see Table 2 and Meylan et al. 2001) the following four parameters relative to G1: the central velocity dispersion $\sigma_{p}(0)=28 \mathrm{~km} \mathrm{~s}^{-1}$, the integrated absolute visual magnitude $M_{V}=-10.94 \mathrm{mag}$, the core radius $r_{c}=$ $0.52 \mathrm{pc}$, and the central surface brightness $\mu(0, V)=13.47 \mathrm{mag} \operatorname{arcsec}^{-2}$. The positions of G1 in the different diagrams defined by Kormendy (1985), using the above four parameters, always put it on the sequence defined by globular clusters, and definitely away from the other sequences defined by elliptical galaxies, bulges, and dwarf spheroidal galaxies (Fig. 1). The same is true for $\omega$ Centauri (Meylan et al. 2001).

Little is known about the positions, in these diagrams, of the nuclei of nucleated dwarf elliptical galaxies, which could be the progenitors of the most massive, if not all, globular clusters (Zinnecker 1988, Freeman 1993). The above four parameters are known only for the nucleus of one dwarf elliptical, viz., NGC 205, and their values put this object, in Kormendy's diagram, close to G1, right on the sequence of globular clusters (see Table 2 and Fig. 1). This result does not prove by itself that all massive globular clusters are the remnant cores of nucleated dwarf galaxies.

At the moment, only the anti-correlation of metallicity with age recently observed in $\omega$ Centauri suggests that this cluster enriched itself over a time scale of about 3 Gyr (Hughes \& Wallerstein 2000 and Hilker \& Richtler 2000). This contradicts the general idea that all the stars in a globular cluster are coeval, and may favor the origin of $\omega$ Centauri as being the remaining core of a larger entity, e.g., of a former nucleated dwarf elliptical galaxy. In any case, by the mere fact that their large masses imply complicated stellar and dynamical evolutions, the very massive globular clusters may blur the former clear (or simplistic) difference between globular clusters and dwarf galaxies.

\section{Mass Estimates of Young Star Clusters}

So far, we have discussed only mass estimates related to old, rich star clusters. HST has triggered numerous studies of young, bright starburst clusters which may be quite massive. See, e.g., Holtzmann et al. (1992) in the case of NGC 1275 clusters, and Schweizer \& Seitzer (1993) and Whitmore et al. (1993) in the case NGC 7252. Conti \& Vacca (1994) use HST/FOC UV imaging of various bright knots in the nuclear starburst region of the Wolf-Rayet galaxy H2 2-10. The luminosities of the knots are compared to those predicted from stellar population synthesis models for ages between 1 and $10 \mathrm{Myr}$, from which they estimate the masses of the knots to be between $10^{5}$ and $10^{6} \mathcal{M}_{\odot}$. In a more direct way, Böker et al. (1999) measure a velocity dispersion $\sigma_{o b s}=33 \pm 3 \mathrm{~km} \mathrm{~s}^{-1}$ in the nuclear star cluster of the face-on giant Scd spiral galaxy IC 342 and deduce, by fitting the central surface brightness profile, a cluster mass $\mathcal{M}_{\text {tot }}=6 \times 10^{6} \mathcal{M}_{\odot}$. They infer a best-fitting cluster age in the range $10-60 \times 10^{6} \mathrm{yr}$.

It is worth emphasizing that mass estimates of young star clusters are more difficult to obtain and more uncertain than those of older clusters. This is due to the frequent lack of velocity dispersion measurement and because of the large uncertainties inherent in the use of stellar population synthesis models for young stellar systems whose luminosities evolve quickly (see, e.g., Bruzual 2001). 


\section{The Slow Destruction of Star Clusters}

Numerical works have demonstrated that continual two-body relaxation within globular clusters combined with weak tidal encounters between globular clusters and the Galactic disk and/or bulge will lead, around each globular cluster, to the development of both a halo of unbound stars and tidal tails. All clusters observed, which do not suffer from strong observational biases, present tidal tails (Grillmair et al. 1995, Leon et al. 2000). These tidal tails exhibit projected directions preferentially aligned with the cluster orbit or towards the Galactic center, betraying their recent dynamical evolution through disk and/or bulge shocking. See also Odenkirchen et al. (2001).

Recent theoretical work corroborate these observations. In N-body simulations of globular clusters moving in the Galactic potential well, Combes et al. (1999) observe that once the particles (stars) are unbound, they slowly drift along the globular cluster path and form two huge tidal tails. A cluster is always surrounded by two giant tidal tails and debris, in permanence along its orbit. The length of these tidal tails is of the order 5 tidal radii or more. The orientation of these tidal tails is the signature of the last disk crossing and can constrain strongly the cluster orbit and the Galactic model. Each disk/bulge crossing may extract up to about $1 \%$ of the total mass of the star cluster, leading slowly but surely to its complete evaporation. The lighter the cluster and the stronger the tidal shocks, the faster the destruction process.

\section{Do Globular Clusters Have A Dark Halo?}

Are globular clusters the most massive stellar systems without non-baryonic dark matter? Since both the velocity dispersion profile and the rotation curve of $\omega$ Centauri decrease towards the outer parts of this cluster, we may conclude that there is no dynamical evidence of any massive halo made of non-baryonic dark matter.

In order to investigate further this point among the most poorly studied Galactic clusters, Côté et al. (2001) have obtained KECK/HIRES high-accuracy radial velocities of about 20 stars in six outer-halo globular clusters, localed between 20 and $100 \mathrm{kpc}$ from the center of our Galaxy. The velocity dispersions range between $\sigma_{o b s}=1$ and $5 \mathrm{~km} \mathrm{~s}^{-1}$, with corresponding $\mathcal{M} / L_{V}$ values between 1 and 4 . This is exactly what is expected from the nearby well studied globular clusters such as $\omega$ Centauri and 47 Tucanae.

However there is one single, although conspicuous, exception in the sample of Côté etal. (2001): Pal 13 exhibits a velocity dispersion $\sigma_{o b s}=2.6 \pm 0.3$ $\mathrm{km} \mathrm{s}^{-1}$. With its low and uncertain total luminosity, Pal 13 has a corresponding $\mathcal{M} / L_{V}$ in the range $10<\mathcal{M} / L_{V}<40$. This is quite unique for a globular cluster. Simulations show that such a result would be mimicked, with difficulties, only by an uncomfortably large fraction of binary stars. Binaries exist in globular clusters, but not in large quantities. We should apply Ockham's razor before invoking the presence of a massive dark halo. Such a high $\mathcal{M} / L_{V}$ value could be an indication of a cluster in the late phases of its dynamical evolution, when a large fraction of its total mass is made of white dwarfs, as predicted by Vesperini \& Heggie (1997). It could also be explained by a velocity dispersion inflated 


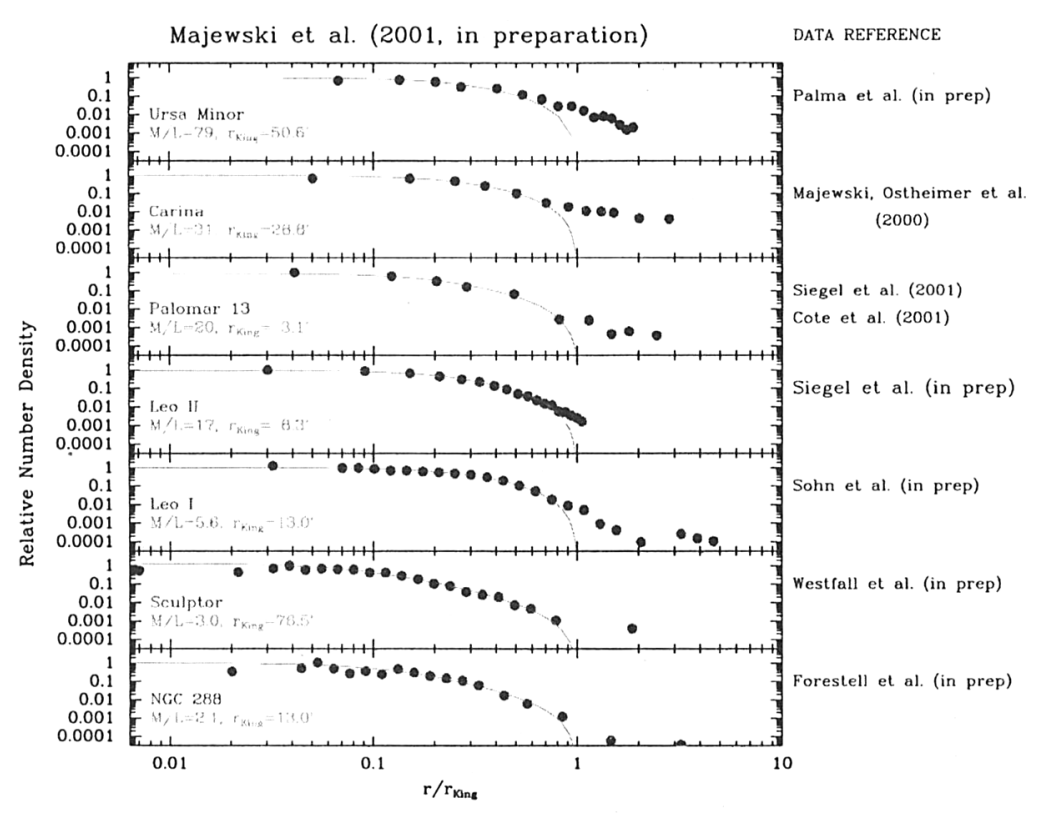

Figure 2. From bottom to top, a sequence of increasingly disturbed surface brightness profiles (two globular clusters and five $\mathrm{dSph}$ galaxies) corresponding to increasing $\mathcal{M} / L_{V}$ values.

because of lack of Virial equilibrium: Pal 13 seems to be in the advanced stages of tidal disruption, a status supported by the recent observations of extra-tidal extension around this cluster (Siegel et al. 2001 and Côté et al. 2001). These two explanations do not exclude each other.

\section{Is Tidal Disruption an Ubiquitous Phenomenon?}

Leon et al. (2000) show that any Galactic globular cluster observed which does not suffer from strong observational biases (e.g., intervening absorption by Galactic cirrus along the line-of-sight) displays a pair of tidal tails. The tidal disturbance may also be unveiled through its effect on the surface brightness profile. An isolated cluster suffering no tidal shock will have a normal King-model profile characterised by a core surrounded by an envelope with a steep profile. Any tidally-perturbed cluster will have an envelope profile departing from the Kingmodel profile as if there were a very strong background of stars: the stronger the tidal effect, the higher the level of the background (i.e., the higher the de- 
parture from King-model profile), which is made of stars escaping the cluster gravitational attraction.

When observing a tidally perturbed globular cluster, there is a high probability of measuring radial velocities of escaping stars, which are no more in Virial equilibrium with the cluster. Such stars would inflate the measured velocity dispersion. The stronger the tidal disturbance, the higher the inflated velocity dispersion, and the larger the mass-to-light ratio. This is exactly what is observed in Fig. 2, from Majewksi et al. (2001), which displays the surface brightness profiles of two globular clusters (NGC 288 with $\mathcal{M} / L_{V}=2.1$ and Pal $13 \mathcal{M} / L_{V} \sim 20$ ) and five dSph galaxies (Sculptor with $\mathcal{M} / L_{V}=3.0$, Leo I with $\mathcal{M} / L_{V}=5.6$, Leo II with $\mathcal{M} / L_{V}=17$, Carina with $\mathcal{M} / L_{V}=31$, and Ursa Major with $\mathcal{M} / L_{V}=79$ ). From the bottom panel (NGC 288) to the top one (Ursa Major) we observe a sequence of increasingly disturbed profiles, with higher and higher departures from the King models, with the most disturbed profile being for Ursa Major. Interestingly, this sequence of increasingly disturbed profiles correspond to a sequence of increasing mass-to-light ratios, as if the $\mathcal{M} / L_{V}$ values were a direct measure of the intensity of the tidal shocks. Is the evidence for dark matter in dSph galaxies evaporating with their stars?

Acknowledgments. It is a pleasure to thank Steve Majewksi (Virginia) and my collaborators Patrick Côté (Rutgers) and George Djorgovski (Caltech) for allowing me to present results in advance of publication. I also thanks Jennifer Lotz (JHU), Roeland van der Marel (STScI), and Brad Whitmore (STScI) for interesting discussions and information about NGC 205.

\section{References}

Anderson J., 1997, Ph.D. thesis, University of California, Berkeley

Anderson J., King I.R., Meylan G., 1998, BAAS, 30, 1347

Anderson J., King I.R., 2000, PASP, 112, 1360

Baumgardt H., 1998, A\&A, 330, 480

Baumgardt H., 2001, in ASP Conf. Ser. Vol. ???, Modes of Star Formation and the Origin of Field Populations, ed. E.K. Grebel and W. Brandner (San Francisco: ASP), in press

Böker T., van der Marel R.P., Vacca W.D., 1999, AJ, 118, 831

Bruzual G.A., 2001, in XI Canary Islands Winter School of Astrophysics, Galaxies at High Redshift, ed. I. Pérez-Fournon and F. Sánchez, (Cambridge: Cambridge Contemporary Astrophysics), in press

Combes F., Leon S., Meylan G., 1999, A\&A, 352, 149

Conti P.S., Vacca W.D., 1994, ApJ, 423, L97

Côté P., Djorgovski S.G., Meylan G., Castro S., McCarthy J.K., 2001, AJ, submitted

Dejonghe H., Merritt D., 1992, ApJ, 391, 531

Dubath P., Meylan G., Mayor M., Magain P., 1990, A\&A, 239, 142

Freeman K.C., 1993, in IAU Symp. 153, Galactic Bulges, ed. H. Dejonghe \& H.J. Habing (Dordrecht: Kluwer), 263 
Freeman K.C., Norris J.E., 1981, ARA\&A, 19, 319

Grillmair C. J., Freeman K. C., Irwin M., 1995, AJ, 109, 2553

Gunn J.E., Griffin R.F., 1979, AJ, 84, 752

Hilker M., Richtler T., 2000, A\&A, 362, 895

Holtzmann J.A., et al. 1992, AJ, 103, 691

Hughes J., Wallerstein G., 2000, AJ, 119, 1225

Kormendy J., 1985, ApJ, 295, 73

Leon S., Meylan G., Combes F., 2000, A\&A, 359, 907

Majewksi S.R., et al., 2001, in preparation

Meylan G., 1988, ApJ, 331, 718

Meylan G., Heggie D.C., 1997, A\&AR, 8, 1-143

Meylan G., Mayor M., Duquennoy A., Dubath P., 1995, A\&A, 303, 761

Meylan G., Sarajedini A., Jablonka P., et al., 2001, AJ, in press

Merritt D., 1993, ApJ, 413, 79

Merritt D., 1996, AJ, 112, 1085

Merritt D., Meylan G., Mayor M., 1997, AJ, 114, 1074

Norris J.E., Freeman K.C., Mayor M., Seitzer P., 1997, ApJ, 487, L187

Odenkirchen M., Grebel E.K., et al., 2001, ApJ, 548, L165

Ogorodnikov K.F., Nezhinskii E.M., Osipkov L.P., 1976, Sov. Astr. Lett. 2, 57

Schweizer F., Seitzer P., 1993, ApJ, 417, L29

Siegel M.H., Majewski S.R., Cudworth K.M., Takamiya M., 2001, AJ, 121, 935 van Leeuwen F., Le Poole R.S., Reijns R.A., Freeman K.C., de Zeeuw P.T., 2000, A\&A, 360, 472

Vesperini E., Heggie D.C., 1997, MNRAS, 289, 898

Whitmore B.C., Schweizer F., Leitherer C., Borne K., Robert C., 1993, AJ, 106, 1354

Zhang Q., Fall S.M., 1999, ApJ, 527, L81

Zinnecker H., Keable C.J., Dunlop J.S., Cannon R.D., Griffiths W.K., 1988, in IAU Symp. 126, Globular Cluster Systems in Galaxies, ed. J.E. Grindlay, A.G.D. Philip, (Dordrecht: Kluwer), 603

\section{Discussion}

T. Armandroff: You raised the possibility of tidal disruption leading to the large velocity dispersion in Ursa Minor. Models for the disruption of dSphs (Piatek \& Pryor, 1995, AJ, 109, 1071; Oh et al., 1995, ApJ, 442, 142) predict a kinematic signature that resembles a rotation curve, as opposed to simply an increased dispersion. Large samples of radial velocities in Draco and Ursa Minor have not shown evidence for the kinematic signature of disruption. Do you have evidence for Ursa Minor or other dSphs matching the modeling?

G. Meylan: The two papers you mention present results of N-body simulations which reach conclusions different from those obtained from other, more recent 
N-body simulations (Kroupa, 1997, New Astron, 2, 139; Klessen \& Kroupa, 1998, ApJ, 498, 143). Kroupa's work shows that dSphs are obtained under the extreme hypothesis that $\mathrm{dSph}$ progenitors are not dominated by dark matter but are significantly shaped by tides through many periastron passages; Piatek \& Pryor simulated only one passage, and Kroupa's results are completely consistent with their findings for one passage only. There are now in Draco photometric observations of stars beyond its measured tidal boundary (Piatek et al., $2001 \mathrm{AJ}, 121,841)$. It will be essential to obtain, for a few of these dSphs, samples of thousands of stellar radial velocities. At the moment, I simply find the correlation between tidal disturbance intensity and $\mathcal{M} / L$ values rather intriguing. This definitely calls for more studies.

G. Da Costa: Comment: Integrated spectrum of nucleus of NGC 205 is A-type, so its stellar population is very different from globular clusters like G1. Question: What is the core radius of the NGC 205 nucleus and does if fit a King model? (different from say M32 with its power-law cusp).

G. Meylan: About the comment: Yes, the stellar populations in the core of NGC 205 are younger than those in G1. This means that the position, in the panels defined by Kormendy (see Fig. 1 above), of a dynamical system older than about 1 Gyr does not depend strongly on its age. Answer to the question: The core radius of the nucleus NGC 205 is 100 mas $=0.35$ pc (Jones et al., 1996, ApJ, 466, 742), similar to a very dense globular cluster easily fitted by a King model. The star cluster in the center of NGC 205 is very different from the nucleus of M32, which has, e.g., a much larger velocity dispersion $\sigma_{o b s}=150$ $\mathrm{km} \mathrm{s}^{-1}$ (Joseph et al., 2001, ApJ, 550, 668). See also Meylan et al. (2001).

C. Grillmair: Two comments: 1) The onset of tidal effects (e.g., break from normal profile to power-law tidal tail, velocity dispersion increase) can depend significantly on the orbital phase of the cluster (strongest effect at apogalacticon). 2) Ben Moore (1996, ApJ, 461, L13) put an upper limit of $\mathcal{M} / L$ of NGC $7089 \equiv \mathrm{M} 2$, based on existence of tidal tails.

E. Wenderoth: Around 15 years ago, the idea that $\omega$ Centauri is a merger was proposed. Since then, is there more evidence to support this idea or to reject it?

G. Meylan: There was a paper by Icke \& Alcaino, 1988, A\&A, 204, 115, suggesting that $\omega$ Centauri could be the result of a merger, this in order to explain (i) its spread in metallicity, (ii) its strong flattening and (iii) its large mass. There is now more evidence about the intrinsic complexity of this globular cluster, as mentioned above in Section 6, although none of these observational facts supports the merger scenario over the other two alternatives. If merger there was, it must have been very early in the life of the two merging proto-cluster clouds, since the mere encounter of two current globular clusters would not induce a merger, except in the case of orbital-parameter adjustment with vanishingly small probabilities. 